\title{
Type 2 Diabetes Decompensation as the Clinical Presentation of Thyroid Storm - Cause or Consequence?
}

\author{
Ana Margarida Monteiro, Cláudia Matta-Coelho, Vera Fernandes and Olinda Marques \\ Endocrinology Department, Hospital de Braga, Braga, Portugal
}

DOI: https://doi.org/10.17925/EE.2017.13.02.99

\begin{abstract}
$\mathrm{T}$ his case study aims to discuss the unusual forms of hyperthyroidism presentation, the nonspecific symptoms and precipitating events. A 70-year-old male was taken to the emergency department for hyperglycaemia, nausea, vomiting and altered mental status with a week of evolution. He had a past medical history of type 2 diabetes, hypertension and dyslipidemia. He had no history of any recent intercurrent illness or infection. At the emergency room, besides hyperglycaemia, ketonemia and slightly elevated C-reactive protein, the basic laboratory panel workup was normal, as was the head computed tomography. He was admitted for metabolic compensation and to study the altered neurological status. During hospitalisation, despite the good glycemic control, he had no improvements in neurological status. At day four of hospitalisation, thyrotoxicosis with thyroid storm criteria was diagnosed. He started on adequate treatment with complete clinical recovery. The associated morbidity and mortality of thyroid storm requires immediate recognition and treatment. Elderly patients are frequently misdiagnosed or diagnosed later due to fewer and less pronounced signs and symptoms.
\end{abstract}

\section{Keywords}

Thyroid storm, thyrotoxicosis, Graves' disease, type 2 diabetes, diabetic ketoacidosis, elderly

Disclosure: Ana Margarida Monteiro, Cláudia MattaCoelho, Vera Fernandes and Olinda Marques have nothing to declare in relation to this article. The authors have not received grants or scholarships. No funding was received in the publication of this article.

Compliance with Ethics: All procedures were followed in accordance with the responsible committee on human experimentation and with the Helsinki Declaration of 1975 and subsequent revisions, and informed consent was received from the patient involved in this case study.

Authorship: All named authors meet the International Committee of Medical Journal Editors (ICMJE) criteria for authorship of this manuscript, take responsibility for the integrity of the work as a whole, and have given final approval to the version to be published. open Access: This article is published under the Creative Commons Attribution Noncommercial License which permits any non-commercial use, distribution, adaptation and reproduction provided the original author(s) and source are given appropriate credit.

Received: 11 May 2017

Accepted: 29 June 2017

Citation: European Endocrinology, 2017;13(2):99-101

Corresponding Author: Ana Margarida Monteiro Endocrinology Department of Hospital de Braga, Sete Fontes - São Victor, 4710-243 Braga, Portugal. E: anamargaridacmonteiro@gmail.com
In contrast to the classical symptoms and obvious signs of a hypermetabolic state, elderly patients present with fewer and less pronounced symptoms such as fatigue, weakness, depression or relative apathy. Moreover, the symptoms are often masked by ageing-associated diseases. ${ }^{1-3}$ Thyroid storm (TS) is a life-threatening exacerbation of hyperthyroidism that requires emergent treatment. This condition is manifested by the decompensation of multiple organs, which is often triggered by severe stress, such as intercurrent illness or perioperative event. ${ }^{3}$ Diagnosis is clinical and is based on the presence of hyperthyroidism in a patient with severe and life-threatening manifestations. To make the diagnosis, Burch and Wartofsky proposed a scoring system modified by Akamizu and colleagues. The treatment goals are reduction of the thyroid hormone synthesis and secretion of thyroid hormones, control of its peripheral effects, resolution of systemic manifestations and treatment of precipitating illness. ${ }^{14,5}$ We report a rare diagnosis of apathetic thyroid storm masked by hyperglycaemia and altered mental status.

\section{Case presentation}

A 70-year-old male was taken to the emergency department for hyperglycaemia, nausea and vomiting with a week of evolution. He also presented asthenia, anorexia, dysphagia, psychomotor retardation and generalised decrease in muscle strength. He had no history of any recent intercurrent illness or infection.

He had type 2 diabetes with a previous glycated hemoglobin of $8.8 \%$. He also had hypertension, dyslipidemia and central retinal thrombosis. He was on detemir and aspartic insulins (100 units/ daily), vildagliptin $100 \mathrm{mg}$, lisinopril $20 \mathrm{mg}$, chlorthalidone $50 \mathrm{mg}$, atorvastatin $40 \mathrm{mg}$, clopidogrel $75 \mathrm{mg}$ and acetylsalicylic acid $100 \mathrm{mg}$. His wife, who managed his medication, denied omission of insulin administration.

On physical examination, he presented signs of dehydration. He was conscious but with temporal disorientation and slowed speech. He had postural tremor. The assessment of muscular strength and visual fields were normal and plantar reflexes were present. The cardiopulmonary auscultation was normal. Body temperature was $36.9^{\circ} \mathrm{C}$, blood pressure 160/85 mmHg and heart rate 100 beats per minute. Capillary glycaemia (388 mg/dl) and blood ketones $(3.8 \mathrm{mmol} / \mathrm{L})$ were high.

On admission, the arterial blood gas revealed a plasma bicarbonate level of $17.7 \mathrm{mEq} / \mathrm{L}$ (NR: 21.0-26.0), pH 7.4 (NR: 7.37-7.45) and anion gap of $25.6 \mathrm{mEq} / \mathrm{L}$ (NR: 8.0-16.0). The serum plasma glucose level was $408 \mathrm{mg} / \mathrm{dl}$ and the calculated plasma osmolarity $342 \mathrm{mOsm} / \mathrm{L}$. The laboratory workup are described in Table 1. The electrocardiogram showed sinus tachycardia. 
Table 1: Laboratory workup at admission

\begin{tabular}{|l|l|l|}
\hline & Results & Reference range \\
\hline Glucose (mg/dL) & 408 & $<126$ \\
\hline Plasma osmolarity (mosm/L) & 342 & $275-295$ \\
\hline Creatinine (mg/dL) & 1.3 & $0.7-1.2$ \\
\hline Urea (mg/dL) & 107 & $15-39$ \\
\hline Sodium (mmol/L) & 141 & $136-145$ \\
\hline Potassium (mmol/L) & 4.7 & $3.5-5.1$ \\
\hline C-reactive protein (mg/L) & 25.6 & $<3.0$ \\
\hline Haemoglobin (g/dL) & 13.0 & $13.5-17.0$ \\
\hline Leukocytes (uL) & 6,400 & $4,000-10,500$ \\
\hline Platelets (uL) & 162,000 & $150,000-400,000$ \\
\hline AST (U/L) & 31 & $15-37$ \\
\hline ALT (U/L) & 76 & $12-78$ \\
\hline
\end{tabular}

$A L T=$ alanine transaminase $; A S T=$ aspartate transaminase

The head computed tomography scan revealed no acute haemorrhagic or ischaemic lesions. However, discrete signs of ischaemic leukoencephalopathy were described.

He started on intravenous fluids and insulin and after 6 hours of continuous insulin perfusion, he achieved glycaemic control with resolution of ketosis and started on subcutaneous insulin. He was hospitalised for metabolic compensation and to study neurological changes.

During hospitalisation, he achieved good glycemic control without improvements in neurological status. On day four of hospitalisation, he was extremely lethargic, and had a fever $\left(38.5^{\circ} \mathrm{C}\right)$, profuse sweating and nausea. The heart rate was $105 \mathrm{bpm}$. There was no clinical or analytical evidence of infection and blood cultures were negative. The laboratory workup evaluation revealed thyrotoxicosis, suppressed thyroidstimulating hormone (TSH) (<0.005 uUI/ml; NR: 0.358-3.74) with elevated free T4 (5.76 ng/dl; NR: 0.76-1.46) and free T3 (6.63 pg/ml; NR: 2.18-3.98). He had elevated anti-thyroid peroxidase antibody (TPOAb) (247 Ul/ml; NR $<35$ ) and positive TSH receptor antibody (20 U/L; NR <9). Based on the Burch and Wartofsky score, thyroid storm was diagnosed (see Table 2). ${ }^{5}$

He immediately started treatment with hydrocortisone (loading dose of $200 \mathrm{mg}$ followed by $100 \mathrm{mg}$ every 8 hours), propranolol (80 mg every 8 hours) and thiamazole ( $20 \mathrm{mg}$ every 6 hours). Lugol solution (8 drops every 6 hours) was initiated one hour after thiamazole. Intravenous fluids and paracetamol were also administrated.

Hydrocortisone, propranolol, Lugol solution and thiamazole were tapered gradually and free $\mathrm{T} 4$ and $\mathrm{T} 3$ became normal after 12 days of therapy. He maintained treatment with thiamazole, with a discharged dose of $10 \mathrm{mg}$ three-times daily.

The cervical ultrasound showed a normal volume thyroid gland with an accentuated and diffusely heterogeneous parenchyma. There were no cervical adenomegalies. Clinical and analytical improvement were noted after therapy and two weeks later he was completely recovered of his neurologic alterations. The metabolic control of diabetes had improved significantly.

Months after the treatment, the thyroid scintigraphy demonstrated a globose gland with diffusely and heterogeneous increased
Table 2: Diagnostic criteria of thyroid storm based on Burch and Wartofsky score

\begin{tabular}{|c|c|}
\hline Criteria & Points \\
\hline \multicolumn{2}{|l|}{ Temperature } \\
\hline $38.5^{\circ} \mathrm{C}$ & 15 \\
\hline \multicolumn{2}{|l|}{ Central nervous system } \\
\hline Lethargy & 20 \\
\hline \multicolumn{2}{|l|}{ Gastrointestinal-hepatic dysfunction } \\
\hline Nausea & 10 \\
\hline \multicolumn{2}{|l|}{ Cardiovascular dysfunction } \\
\hline Tachycardia (105 bpm) & 5 \\
\hline \multicolumn{2}{|l|}{ Heart failure } \\
\hline Absent & 0 \\
\hline \multicolumn{2}{|l|}{ Precipitant history } \\
\hline Absent & 0 \\
\hline Total & 50 \\
\hline
\end{tabular}

radiopharmaceutical uptake. The definitive remission of Graves' disease was achieved after treatment with radioactive iodine (15 mCi). He developed hypothyroidism and he is currently on levothyroxine 137 ug/daily.

\section{Discussion}

Poudel et al. described a case of an 84-year-old woman with type 2 diabetes who developed apathetic hyperthyroidism due to Graves' disease, although without thyroid storm at presentation. ${ }^{\circ} \mathrm{A}$ case of thyroid storm associated with Graves' disease masked by diabetic ketoacidosis in a 59-year-old woman was described by Osada et al. However, in contrast with our case, the patient had no history of diabetes.? In fact, in the published literature, this is the first case of an elderly man with type 2 diabetes that developed apathetic hyperthyroidism culminating in thyroid storm as a first presentation of Graves' disease.

Apathetic hyperthyroidism was first described by Lahey in $1931 .{ }^{8}$ It is most frequently observed in middle-aged and elderly populations and is estimated to occur in 10-15\% of older individuals with hyperthyroidism, although it can be underdiagnosed. The pathogenesis of apathetic hyperthyroidism is unclear. A previous report indicated it may be due to a decrease in adrenergic tone and age-related changes in the autonomic nervous system and resistance of tissues to the effects of thyroid hormone. ${ }^{9.10} \mathrm{~A}$ rapid rate of increase in serum thyroid hormone levels, an increased responsiveness to catecholamines, or an enhanced cellular response to thyroid hormone have been proposed as mechanisms to the development of thyroid storm. However, it remains unclear why certain factors result in the development of thyroid storm. The excess of thyroid hormone is typically not more pronounced than that seen in patients with uncomplicated thyrotoxicosis. ${ }^{11}$ Thyroid storm can occur in patients with long-standing untreated hyperthyroidism but it is often precipitated by an acute event such as thyroid or nonthyroidal surgery, trauma, infection, an acute iodine load, labor or irregular use or discontinuation of antithyroid drugs. Other conditions known to be associated include cytotoxic chemotherapy, aspirin overdose, ketoacidosis or organophosphate intoxication. ${ }^{12}$ In the present case, hyperglycaemia was the only precipitating factor identified, however, it might be a consequence of thyrotoxicosis. Several mechanisms explains the hyperglycaemia in hyperthyroidism, including a diminished insulin half-life secondary to an increased rate of degradation and a higher release of biologically 
inactive insulin precursors. Also, there is an increase in glucose gut absorption mediated by the excess of thyroid hormones and an enhanced endogenous production of glucose. ${ }^{13}$ We may conclude that the triggering factor remains unknown.

Thyroid storm can happen in hyperthyroidism of any cause but the most common etiology is an underlying Graves' disease..$^{14}$ The referred patient had been evaluated for thyroid dysfunction in the previous months and he had normal thyroid function. After thyrotoxicosis was diagnosis, we had positivity for anti-TSH receptor antibodies, an ultrasound and a scintigraphy compatible with Graves' disease, an uncommon disease in males and in the elderly. ${ }^{15}$

In the reported case, the unusual form of hyperthyroidism presentation, the nonspecific symptoms that were compatible with multiple equally serious pathologies, and the absence of known thyroid disease and previous normal thyroid function constituted a diagnostic challenge. Moreover, the concomitant diabetic decompensation lasting for several weeks delayed the diagnosis. The definitive response to antithyroid drug, iodine and steroid, supported the diagnosis of thyroid storm. $\square$
1. De Leo S, Lee SY, Braverman LE, Hyperthyroidism, Lancet, 2016;388:906-918

2. Goichot B, Caron P. Landron F, Bouée S, Clinical presentation of hyperthyroidism in a large representative sample of outpatients in France: relationships with age, aetiology and hormonal parameters, Clin Endocrinol (Oxf), 2016:84:445-51.

3. Boelaert K, Torlinska B, Holder RL, Franklyn JA, Older subjects with hyperthyroidism present with a paucity of symptoms and with hyperthyroidism present with a paucity of symptoms a
signs: A large cross-sectional study, I Clin Endocrinol Metab, signs: A large cross-s

4. Akamizu T, Satoh T, Isozaki O, et al., Diagnostic criteria, clinical features, and incidence of thyroid storm based on nationwid surveys, Thyroid, 2012;22:661-79.

5. Burch HB, Wartofsky L, Life-threatening thyrotoxicosis. Thyroid storm, Endocrinol Metab Clin North Am, 1993;22:263-77.

Poudel RR, Belbase B, Belbase B, Kafle NK, Apathetic

thyrotoxicosis presenting with diabetes mellitus, J Community Hosp Intern Med Perspect, 2014;4:25502.

7. Osada E, Hiroi N, Sue M, et al., Thyroid storm associated with ${ }^{\prime}$, with Graves disease covered by di

8. Lahey FH, Apathetic Thyroidism, Ann Surg, 1931;93:1026-30.

9. Kim M, Severe thyrotoxicosis in the elderly, Endotext, 2000. Available at: www.ncbi.nlm.nih.gov/pubmed/25905327 (accessed 7 December 2017).

10. Wu W, Sun Z, Yu J, et al., A clinical retrospective analysis of factors associated with apathetic hyperthyroidism, Pathobiology, 2010;77:46-51.
11. Ross DS, Thyroid storm - UpToDate, 2016. Available at: www.uptodate.com/contents/thyroid-storm?source=search result\&search=thyroid stomr\&selectedTitle=1 150 (accessed 9 January 2017)

12. Klubo-Gwiezdzinska J, Wartofsky L, Thyroid emergencies, Med Clin North Am, 2012;96:385-403.

13. Hage M, Zantout MS, Azar ST, Thyroid disorders and diabetes Hage M, Zantout MS, Azar ST, Thyroid
mellitus, 1 Thyroid Res, 2011:439463.

14. Chiha M, Samarasinghe S, Kabaker AS, Thyroid storm: An updated review, I Intensive Care Med, 2015;30:131-40

15. Marino M, Vitti P, Chiovato L, Graves' Disease. In: Jameson JL, De Groot L, de Kretser DM, et al. (eds), Endocrinology: Adult and Pediatric, 7th ed., Elsevier, 2016;1437-65. 